\title{
The Natural History of Aerosolized Francisella tularensis Infection in Cynomolgus Macaques
}

\author{
Ondraya M. Frick ${ }^{1}$, Virginia A. Livingston ${ }^{1,2}$, Chris A. Whitehouse ${ }^{3,4}$, Sarah L. Norris ${ }^{1}$, Derron A. Alves ${ }^{5,6}$, \\ Paul R. Facemire ${ }^{5}$, Douglas S. Reed ${ }^{7}$ (I) and Aysegul Nalca ${ }^{8, *}$ (i)
}

1 Veterinary Medicine Division, U.S. Army Medical Research Institute of Infectious Diseases (USAMRIID), Frederick, MD 21702, USA; ondraya.m.frick.civ@mail.mil (O.M.F.); virginia.a.livingston6.civ@mail.mil (V.A.L.); sarah.1.norris2.civ@mail.mil (S.L.N.)

2 Naval Medical Research Center, Undersea Medicine Department, Silver Spring, MD 20910, USA

3 Molecular and Translational Sciences Division, U.S. Army Medical Research Institute of Infectious Diseases (USAMRIID), Frederick, MD 21702, USA; Chris.Whitehouse@fda.hhs.gov

4 Office of Research, Center for Veterinary Medicine, U.S. Food and Drug Administration, Laurel, MD 20708, USA

5 Pathology Division, U.S. Army Medical Research Institute of Infectious Diseases (USAMRIID), Frederick, MD 21702, USA; derron.a.alves.mil@mail.mil (D.A.A.); paul.r.facemire2.mil@mail.mil (P.R.F.)

6 Veterinary Services and Public Health Sanitation Directorate, Army Public Health Center, Aberdeen Proving Ground, MD 21010, USA

7 Center for Vaccine Research, University of Pittsburgh, Pittsburgh, PA 15261, USA; dsreed@pitt.edu

8 Core Support Directorate, U.S. Army Medical Research Institute of Infectious Diseases (USAMRIID), Frederick, MD 21702, USA

* Correspondence: aysegul.nalca.civ@mail.mil; Tel.: +1-301-619-8495

Citation: Frick, O.M.; Livingston, V.A.; Whitehouse, C.A.; Norris, S.L.; Alves, D.A.; Facemire, P.R.; Reed, D.S.; Nalca, A. The Natural History of Aerosolized Francisella tularensis Infection in Cynomolgus Macaques. Pathogens 2021, 10, 597. https:// doi.org/10.3390/pathogens10050597

Academic Editor: Bonto Faburay

Received: 3 March 2021

Accepted: 7 May 2021

Published: 13 May 2021

Publisher's Note: MDPI stays neutral with regard to jurisdictional claims in published maps and institutional affiliations.

Copyright: (C) 2021 by the authors Licensee MDPI, Basel, Switzerland. This article is an open access article distributed under the terms and conditions of the Creative Commons Attribution (CC BY) license (https:/ / creativecommons.org/licenses/by/ $4.0 /)$.

\begin{abstract}
Tularemia is a severe, zoonotic infection caused by the Gram-negative bacterium Francisella tularensis. Inhalation results in a rapid, severe bacterial pneumonia and sepsis, which can be lethal. Because the cynomolgus macaque is the accepted nonhuman primate model for tularemia, we conducted a natural history study of pneumonic tularemia by exposing macaques to target inhaled doses of 50, 500, or 5000 colony forming units (CFU) of F. tularensis subsp. tularensis SCHU S4. Two animals within the 50 CFU group (calculated doses of 10 and 11 CFU) survived the challenge, while the remainder succumbed to infection. Exposure of cynomolgus macaques to aerosolized SCHU S4 resulted in fever, anorexia, increased white blood cell counts, lymphopenia, thrombocytopenia, increased liver enzymes, alterations in electrocardiogram (ECG), and pathological changes typical of infection with $F$. tularensis, regardless of the challenge dose. Blood pressure dropped during the febrile phase, particularly as temperature began to drop and macaques succumbed to the disease. ECG analysis indicated that in $33 \%$ of the macaques, heart rate was not elevated during the febrile phase (Faget's sign; pulse-temperature disassociation), which has been reported in a similar percentage of human cases. These results indicated that infection of cynomolgus macaques with aerosolized $F$. tularensis results in similar disease progression and outcome as seen in humans, and that cynomolgus macaques are a reliable animal model to test medical countermeasures against aerosolized F. tularensis.
\end{abstract}

Keywords: Francisella tularensis; tularemia; aerosol; animal model; cynomolgus macaque

\section{Introduction}

Francisella tularensis is an intracellular Gram-negative bacterium and the causative agent of the disease tularemia. Infection generally produces an acute febrile illness; the disease can manifest as a range of possible clinical presentations, largely dependent on the route of infection, dose, virulence of the bacterial strain, and host immune response [1]. After exposure, F. tularensis multiplies at the initial site of infection prior to spreading to the regional lymph nodes, liver, and spleen, and then to other organs [2]. The most common 
form of disease is ulceroglandular, involving an ulcer at the site of entry and regional lymphadenopathy, which is commonly acquired by direct contact with infected animals or arthropod bites [3]. Less commonly, variations of ulceroglandular disease are associated with different sites of entry and include oculoglandular and oropharyngeal disease. The oropharyngeal form results from ingesting contaminated food or water and, while rare, it has been reported with increasing frequency in Turkey and other European countries [4-6]. Pulmonary disease (pneumonic tularemia) can result from clinical progression of the ulceroglandular forms or from direct inhalation of $F$. tularensis organisms. Further clinical progression from any of the forms of tularemia can result in typhoidal tularemia leading to a state of septic shock, organ failure, and death of the host. F. tularensis is highly successful at evading the immune system, principally by escaping the phagosome and replicating in the cytosol of eukaryotic cells, including macrophages and dendritic cells. Dissemination of the microbe to distant sites within the body occurs via trafficking through the regional lymph nodes into the bloodstream [7].

Naturally occurring outbreaks of pneumonic tularemia still occur in the United States, as exemplified by outbreaks on Martha's Vineyard, Massachusetts in the summer of 1978 and again in $2000[8,9]$. The number of cases averaged in the thousands early in the 20th century and has steadily declined since the 1950s, likely a result of increased urbanization of the population. Historically, F. tularensis was studied by Japan (prior to 1945) and the former Soviet Union for its potential as a biological weapon, and it was included in the United States offensive biological weapons program until that program was discontinued in $1969[10,11]$. A WHO study in 1969 found that intentional release of aerosolized F. tularensis in a densely populated area could cause 250,000 cases and 19,000 fatalities in a short timeframe [12]. The organism is currently listed as a Tier 1 select agent by the Federal Select Agent \& Toxins Program (FSAP), those for which there is the most concern about intentional use as a biological weapon [13].

Models of tularemia disease have been evaluated in a variety of animal species, including mice, rabbits, rats, guinea pigs, and monkeys [14]. In a previous study, we compared the $\mathrm{LD}_{50}$ and disease outcome of inhalational tularemia in three nonhuman primate (NHP) species (cynomolgus and rhesus macaques, and African green monkeys) $[15,16]$. Thus, to more extensively characterize the disease progression and pathogenesis in a cynomolgus macaque model, we exposed groups of cynomolgus macaques to three different target doses (50 CFU, 500 CFU, and 5000 CFU) of aerosolized F. tularensis SCHU S4. Based on these data, as well as more comprehensive analysis of studies from multiple facilities, the cynomolgus macaque inhalation model was selected as the nonhuman primate model of the human disease resulting from inhalation exposure to $F$. tularensis. The resulting data from this study contributed to the knowledge base needed for use of this model for evaluation of vaccines and therapeutics for tularemia to satisfy the FDA Animal Rule.

\section{Results}

\subsection{Survival and Clinical Signs}

Three groups of cynomolgus macaques were exposed to increasing doses of aerosolized F. tularensis SCHU S4 (Table 1). The target doses of 50 CFU $(n=10), 500$ CFU $(n=9)$, and $5000 \mathrm{CFU}(n=9)$ were chosen based on F. tularensis SCHU S4 median lethal dose $\left(\mathrm{LD}_{50}\right)$ in cynomolgus macaques previously determined. The calculated inhaled doses were comparable to the target doses for each group (Table 1). Survival was dose-dependent, with the exposures to high doses of $5000 \mathrm{CFU}$ (range of $1177 \mathrm{CFU}$ to $5860 \mathrm{CFU}$ ) and the intermediate dose of $500 \mathrm{CFU}$ (range of 134 to $749 \mathrm{CFU}$ ) resulting in no surviving animals. Exposures to the low dose of 50 CFU (range of 10 CFU to 110 CFU) resulted in survival of 2 out of 10 $(20 \%)$ animals which were exposed to 10 and 11 CFUs (Figure 1). There were significant differences in the survival curves when comparing the low dose group to the intermediate $(p=0.0324)$ and high dose groups $(p=0.0036)$ (Table 2$)$. With the exception of one animal in the intermediate dose group, the majority of macaques receiving high and intermediate doses met criteria for euthanasia between days 6 and 8, whereas animals receiving low 
doses had a much wider range, meeting criteria for euthanasia between 7 and 19 days (Table 1). Since most of the animals succumbed to disease on day 10, the following data is showing only up to day 10 post infection pi accurate comparison.

Table 1. Comparison of survival between groups.

\begin{tabular}{|c|c|c|c|c|}
\hline \multirow{2}{*}{$\begin{array}{l}\text { Target Exposure } \\
\text { Dose Groups }\end{array}$} & \multirow{2}{*}{$\begin{array}{c}\text { Average Inhaled } \\
\text { Dose (CFU) }\end{array}$} & \multicolumn{2}{|c|}{ Days to Death * } & \multirow{2}{*}{ Survivals/Total } \\
\hline & & Mean & Std. Dev. & \\
\hline $50 \mathrm{CFU}$ & $49 \pm 32$ & 11.9 & 4.5 & $2 / 10$ \\
\hline $500 \mathrm{CFU}$ & $507 \pm 250$ & 8.4 & 3.4 & $0 / 9$ \\
\hline $5000 \mathrm{CFU}$ & $4047 \pm 1563$ & 7.1 & 0.9 & $0 / 9$ \\
\hline
\end{tabular}

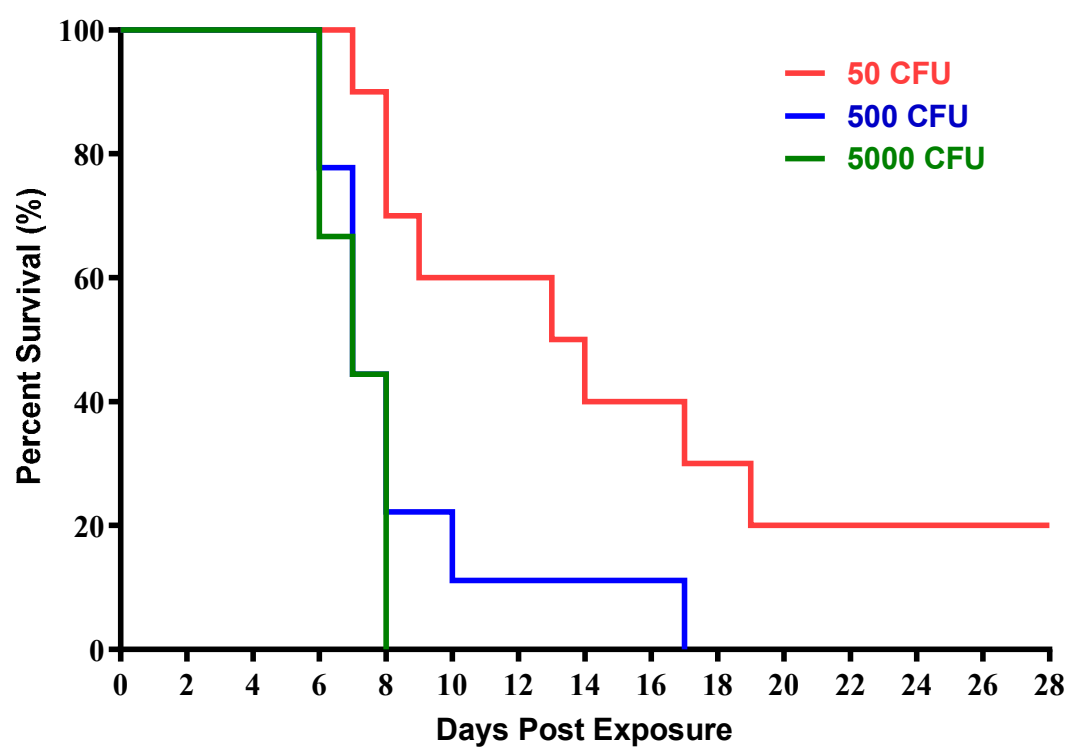

Figure 1. Survival of cynomolgus macaques exposed to increasing doses of aerosolized F. tularensis. Group sizes were $n=10$ (50 CFU target dose), $n=9$ (500 CFU target dose), and $n=9$ (5000 CFU target dose).

Table 2. Survival Comparison ( $p$ values).

\begin{tabular}{ccc}
\hline Comparison & Time-to-Death (Days) & Survival Curve \\
\hline $\mathbf{5 0}$ vs. $\mathbf{5 0 0} \mathrm{CFU}$ & 0.2117 & $\mathbf{0 . 0 3 2 4}$ \\
$\mathbf{5 0}$ vs. $\mathbf{5 0 0 0} \mathrm{CFU}$ & 0.0630 & $\mathbf{0 . 0 0 3 6}$ \\
$\mathbf{5 0 0}$ vs. $\mathbf{5 0 0 0} \mathrm{CFU}$ & 0.2891 & 0.4228 \\
\hline
\end{tabular}

Figure 2 shows temperature data from representative macaques in each dose group. Except for two macaques in the lowest dose group, all macaques exposed to $F$. tularensis in this study developed a fever between one and four days after infection. The onset of sustained ( $6+$ hours) fever was generally earlier in the higher dose group (day 1.8 vs. day 3.4, Table 3), however, the differences in onset of sustained fever between the groups failed to meet criteria for statistical significance $(p=0.0644)$ (Table 2). Fever hours and maximum elevation in body temperature were longer and higher in the high dose group. However, there were no significant differences between groups for fever duration or average elevation (Supplementary Table S1). 

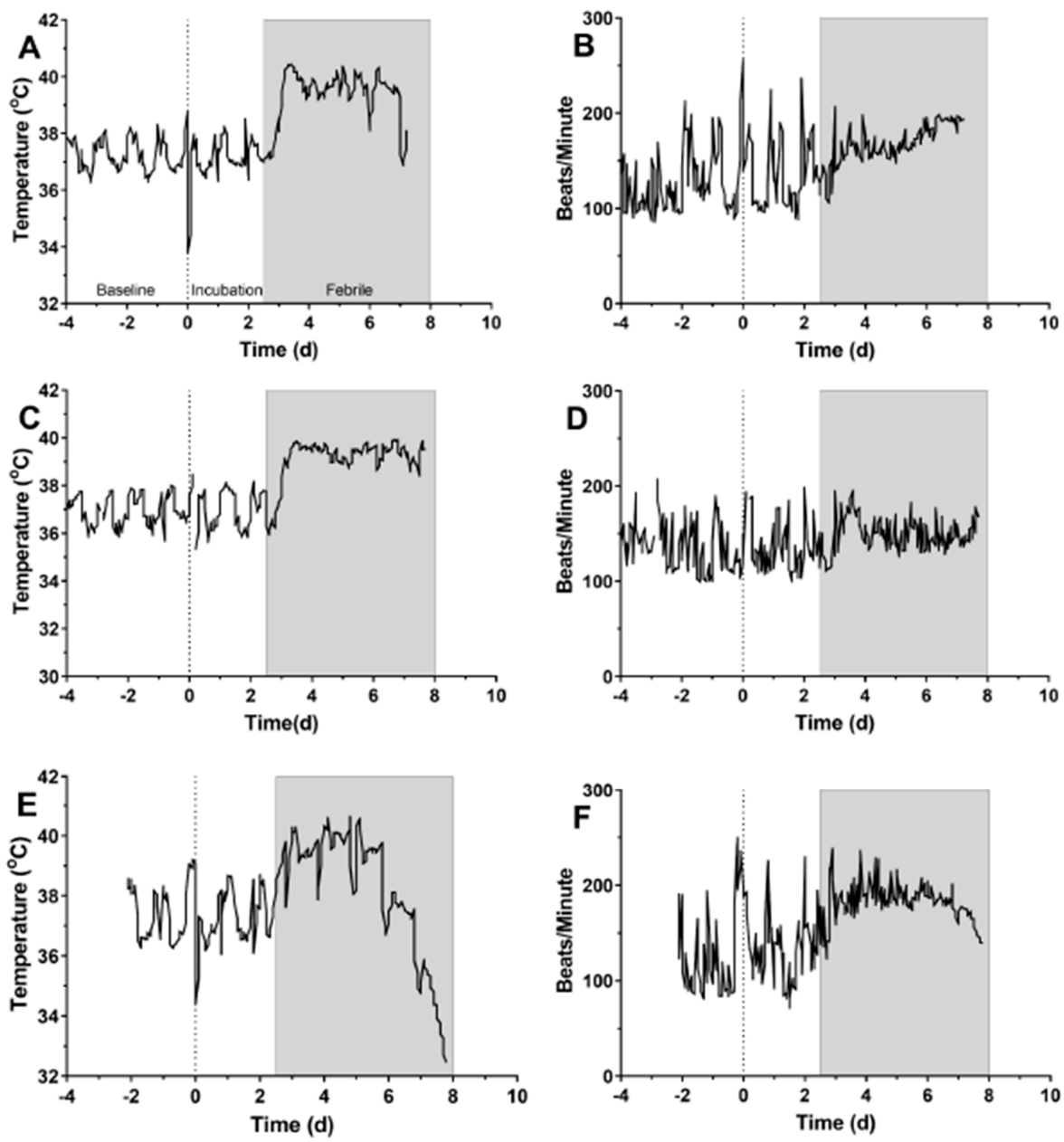

Figure 2. Fever and heart rate changes recorded by telemetry in macaques exposed to aerosolized F. tularensis. Data is shown from representative data from individual macaques in the low (A,B), intermediate (C,D), and high (E,F) dose groups. Data collected prior to infection is considered baseline and is used for prediction of body temperature post-infection (A). The period between infection and fever onset is considered the incubation period. The gray bar represents the febrile period in each macaque to allow comparison with the heart rate data, beginning at 2.5 days post-infection and continuing to day 8 post-infection.

Table 3. Summary of fever data.

\begin{tabular}{|c|c|c|c|c|c|c|c|c|c|}
\hline \multirow[b]{2}{*}{ Variable } & \multicolumn{3}{|c|}{$50 \mathrm{CFU}^{\mathrm{e}}$} & \multicolumn{3}{|c|}{$500 \mathrm{CFU}^{\mathrm{f}}$} & \multicolumn{3}{|c|}{5000 CFU $\mathrm{g}$} \\
\hline & $\mathbf{N}$ & Mean & SD & $\mathbf{N}$ & Mean & SD & $\mathbf{N}$ & Mean & SD \\
\hline Study Day of $6+$ Hours of Fever ${ }^{a}$ & 6 & 3.4 & 1.50 & 5 & 3.4 & 0.11 & 7 & 1.8 & 1.24 \\
\hline Days with $12+$ Hours of Fever ${ }^{b}$ & 6 & 3.5 & 0.84 & 4 & 4.5 & 1.00 & 5 & 3.4 & 1.82 \\
\hline Maximum Change in Temperature & 8 & 4.6 & 1.70 & 8 & 3.8 & 2.04 & 7 & 6.3 & 5.39 \\
\hline Fever Duration (hours) & 8 & 77.5 & 42.85 & 8 & 58.4 & 53.74 & 7 & 74.1 & 47.85 \\
\hline Fever Hours ${ }^{c}$ & 8 & 246.3 & 165.97 & 8 & 226.8 & 268.24 & 7 & 447.9 & 450.46 \\
\hline Average Elevation ${ }^{d}$ & 8 & 3.3 & 1.48 & 8 & 3.0 & 1.56 & 7 & 4.7 & 4.14 \\
\hline
\end{tabular}

${ }^{a}$ Defined as the first day with 6 or more hours of significant temperature elevation (as determined by autoregressive integrated moving average (ARIMA) modeling). ${ }^{\mathrm{b}}$ Calculated as the number of days with 12 or more hours of significant temperature elevation. ${ }^{\mathrm{c}}$ Calculated as the product of each significant increase in temperature elevation (in degrees) multiplied by the length of time of elevation (in hours).

${ }^{\mathrm{d}}$ Calculated by dividing fever hours by fever duration in hours. ${ }^{\mathrm{e}}$ Of the 10 animals in the $50 \mathrm{CFU}$ group, 2 had no telemetry data, and 2 did not have $6+$ hours of sustained fever. ${ }^{\mathrm{f}}$ Of the 9 animals in the 500 CFU group, 1 had no telemetry data, 3 did not have $6+$ hours of sustained fever, and 4 did not have 12+ hours of sustained fever. ${ }^{g}$ Of the 9 animals in the 5000 CFU group, 2 had no telemetry data, and 2 did not have $12+$ hours of sustained fever. 
A subset of macaques were implanted with telemetry devices that monitored ECG and blood pressure in addition to body temperature. Temperature and heart rate data from-three representative macaques from each dose group are shown in Figure 2. In two of the three macaques, there was a clear elevation in heart rate that roughly coincides with fever; in the third macaque, the heart rate was not elevated, although there was an apparent loss in normal diurnal variation. Median heart rates in the baseline, incubation (pre-fever onset) and febrile period were analyzed further for individual macaques; the results are shown in Figure 3 and Table 4. For five of the eight macaques shown in Figure 3, there was a highly significant increase in heart rate in the febrile period; in the remaining three heart rate was not as significantly increased or was not significant at all. Further evaluation of the heart rate data found that only one of thirteen macaques did not have a significantly elevated heart rate in the febrile period (Table 4). Elevation in heart rate roughly correlated with the challenge dose $\left(r^{2}=0.49\right)$. However, the degree of elevation in the febrile period was quite low, with a median percent change of only $21.1 \%$. Six of the thirteen macaques had a less than 20 percent increase in heart rate from baseline. Using Leibermeister's rule of an expected $8 \mathrm{bpm}$ increase in heart rate with every $1{ }^{\circ} \mathrm{C}$ increase in body temperature, the predicted increase in heart rate during the febrile period was calculated and compared with the actual increase in heart rate for each macaque (Table 4). For one macaque, we were not able to predict the increase in heart rate because of insufficient baseline temperature data. Based on the elevation in heart rate between the febrile and baseline periods, it is likely this macaque had a heart rate elevated greater than what was predicted. For eight of the macaques, the actual increase was greater than predicted in the febrile period. For four macaques, however, the actual increase in heart rate during the febrile period was lower than was predicted.

Table 4. Changes in heart rate in individual macaques after infection.

\begin{tabular}{|c|c|c|c|c|c|c|c|c|}
\hline Dose * & Baseline $^{£}$ & Incubation & Febrile & $\begin{array}{l}\text { Percent } \\
\text { Change }\end{array}$ & $p$ & Predicted $\gamma$ & Actual $^{\alpha}$ & Diff. ${ }^{\beta}$ \\
\hline 31 & 137.3 & 127.2 & 163.7 & 19.2 & $<0.0001$ & 20.76 & 26.4 & 5.64 \\
\hline 59 & 134.2 & 141.8 & 178.8 & 33.2 & $<0.0001$ & 18.85 & 44.6 & 25.75 \\
\hline 61 & 119 & 126.4 & 169.2 & 42.2 & $<0.0001$ & 20.07 & 50.2 & 30.13 \\
\hline 72 & 145.9 & 159.5 & 150.7 & 3.3 & 0.043 & 19.68 & 4.8 & -14.88 \\
\hline 551 & 133.4 & 127.5 & 150.1 & 12.5 & $<0.0001$ & 19.31 & 16.7 & -2.61 \\
\hline 636 & 144.3 & 164.1 & 182.4 & 26.4 & $<0.0001$ & 12.85 & 38.1 & 25.25 \\
\hline 639 & 177.6 & 177.7 & 178.9 & 0.7 & 0.861 & 18.86 & 1.3 & -17.56 \\
\hline 728 & 138.2 & 145.6 & 167.4 & 21.1 & $<0.0001$ & 12.89 & 29.2 & 16.31 \\
\hline 749 & 152.5 & 133 & 164.5 & 7.9 & 0.002 & 13.24 & 12 & -1.24 \\
\hline $3977^{\varsigma}$ & 109.4 & 134.8 & 182.8 & 67.1 & $<0.0001$ & & & \\
\hline 4664 & 169.4 & 183.6 & 188.6 & 11.3 & $<0.0001$ & 14.93 & 19.2 & 4.27 \\
\hline 4717 & 143.7 & 155.8 & 185.6 & 29.2 & $<0.0001$ & 12.91 & 41.9 & 28.99 \\
\hline 4894 & 128.7 & 130.1 & 164.4 & 27.7 & $<0.0001$ & 20.04 & 35.7 & 15.66 \\
\hline Median & 138.2 & 141.8 & 169.2 & 21.1 & & 18.9 & 27.8 & 10.6 \\
\hline
\end{tabular}

${ }^{*}$ dose in cfu. ${ }^{£}$ Median beats per minute. ${ }^{\gamma}$ predicted elevation in heart rate, determined by median elevation in temperature. ${ }^{\alpha}$ actual elevation in heart rate in febrile period from baseline. ${ }^{\beta}$ difference between actual and predicted elevation in heart rate. ${ }^{\zeta}$ predicted heart rate could not be calculated for this macaque because of inadequate baseline data for temperature forecasting.

Figure 4A-D shows changes in blood pressure over time for four macaques infected with F. tularensis. Prior to fever onset, there is no notable change in either systolic or diastolic blood pressure. For three of the four, blood pressure drops throughout the febrile period until the macaque succumbed to the infection (Figure $4 \mathrm{~A}-\mathrm{C}$ ). In the fourth animal, diastolic pressure drops in the febrile period, but systolic pressure does not (Figure 4D). There is, however, a notable change in the pattern of systolic pressure with the data overall smoother and less variation during the febrile period. It is notable that of the four shown, this macaque did not have an elevated heart rate. 

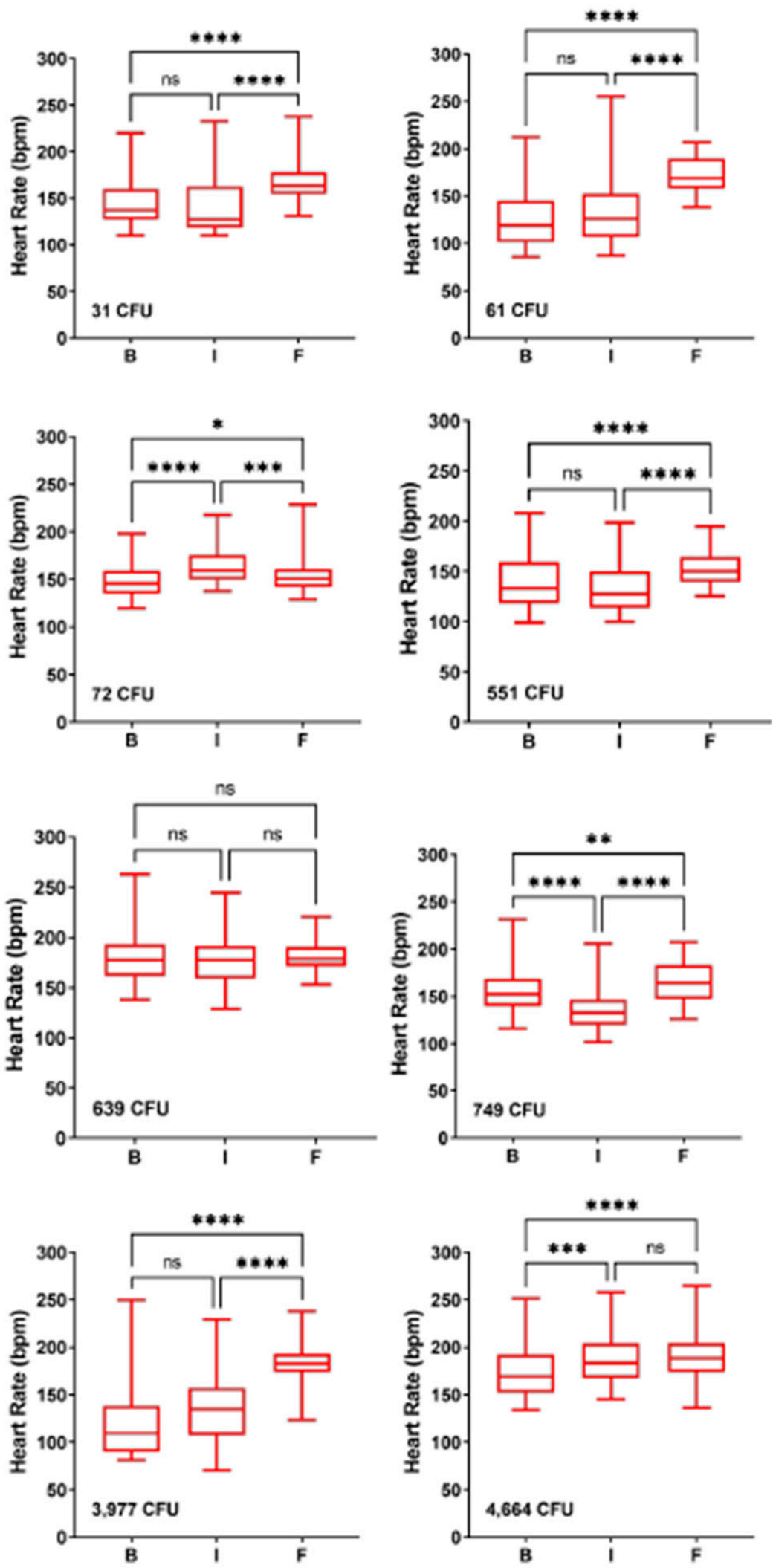

Figure 3. Mild elevation in heart rate during the febrile period. Data is shown from eight individual macaques across all dose ranges; values plotted are the median with the standard deviation and range for each period. Challenge doses are shown for each macaque. Significant changes from baseline were determined by Brown-Forsythe's analysis of variance (ANOVA). n.s. = not significant, ${ }^{*}=p<0.05,{ }^{* *} p<0.01,{ }^{* * *} p<0.001,{ }^{* * * *} p<0.0001$. 

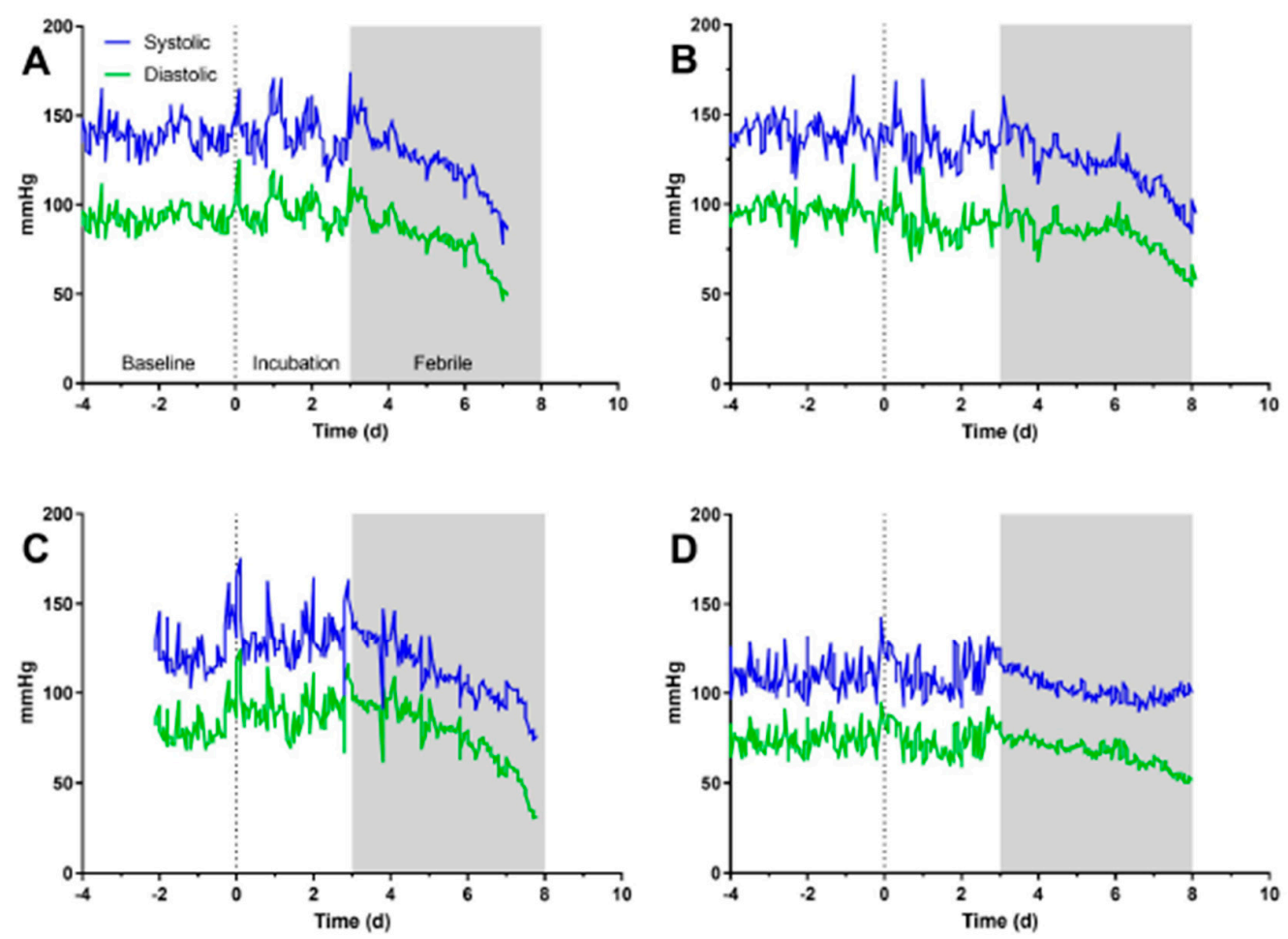

Figure 4. Loss of blood pressure during the febrile period. Graphs shown data for systolic (blue) and diastolic (green) blood pressure recorded by telemetry from four individual macaques (A-D) across all dose ranges. Gray bars on each graph mark the febrile period.

Increases in respiration rate were noted in all groups. While low and intermediate dose group macaques demonstrated increased respiratory rate starting on Day 3, the high dose group had an increased respiratory rate on Day 4. All macaques in the high group dose had gradually increased respiratory rates until they were euthanized (Figure 5A). Clinical signs noted included increased body temperature, elevated white blood cell (WBC) count, and increased respirations. Overall, the onset of increase in clinical score was earliest in the high dose group on Day 2 post-exposure with decreased appetite (Figure 5B). Over half of all macaques developed a cough between Days 7 and 9 with the greatest observed numbers in the high dose group $(78 \%)$. Loose stool was observed primarily in the low dose group and in $25 \%$ of macaques overall. Body weights of all macaques were taken on Day-1 and on the day of euthanasia. The high and intermediate dose groups showed similar weight loss of $8.3 \%(p=0.0171)$ and $8.7 \%(p=0.0171)$, respectively, whereas the low dose group lost an average of $2.4 \%$ (data not shown). Disease scores for all groups exposed to F. tularensis peaked on Day 8 post-exposure and the last members of the high dose group macaques were euthanized on this day.

A)

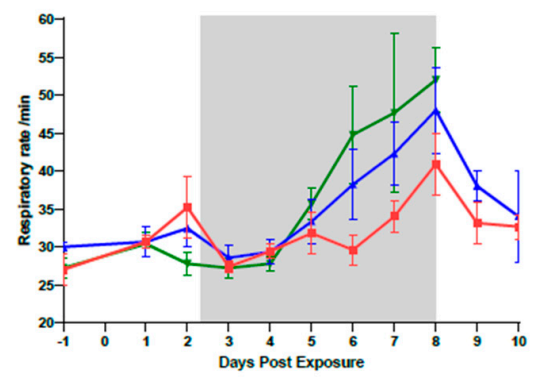

B)

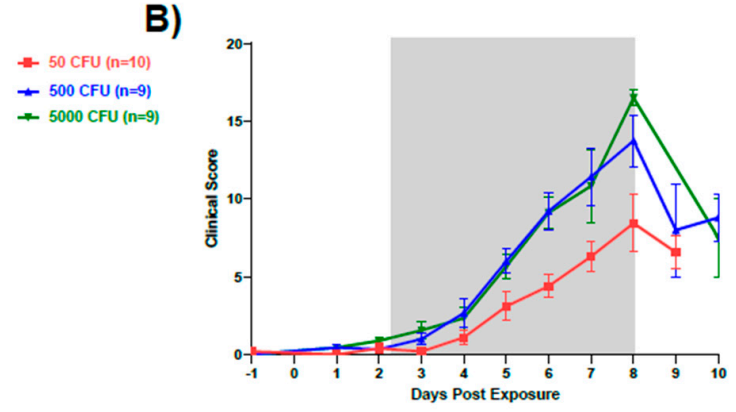

Figure 5. Changes in respiration and clinical score after infection. Graphs show changes in (A) average respiration rate and (B) average clinical score for each dose group. Gray bars on each graph mark the febrile period. 


\subsection{Clinical Laboratory Evaluation}

Changes in $\mathrm{CBC}$ s and blood chemistries were observed in all groups after exposure to $F$. tularensis. Similar to our observations in the previous published study, WBC counts increased starting Day 2 for the high and low dose group and Day 3 for the intermediate dose group (Figure 6). Decrease in total protein and albumin (ALB) values observed by Day 3 (Figure 7A,B). There were no significant differences in total protein between the three groups. In contrast, albumin values were significantly lower in the intermediate $(p=0.0003)$ and high dose groups $(p=0.0014)$ compared to the low dose group. In addition, both intermediate and high dose group values were significantly lower than the normal range $(p<0.05)$. Liver enzymes aspartate transaminase (AST), alanine transaminase (ALT), and alkaline phosphatase (ALKP) were elevated in all groups (Figure 7C-E). Pairwise comparisons showed significant differences between the low and high dose groups for AST, ALT, and ALKP ( $p=0.0062, p=0.0118$, and $p<0.0001$, respectively). The greatest increases outside of the normal range were observed in elevated ALKP values for the high dose group on Days $6(p<0.001), 7(p<0.01)$ and $8(p<0.001)$. Levels of LDH (lactate dehydrogenase) increased starting on Day 4 for all groups with the high dose group showing the greatest increases in comparison to the low dose group $(p=0.0385)$ (Figure 7F). Except the highest dose group, blood urea nitrogen (BUN) levels stayed in normal limits; similarly, creatinine (CRE) levels remained within a low / normal range (Figure 7G,H).

A)

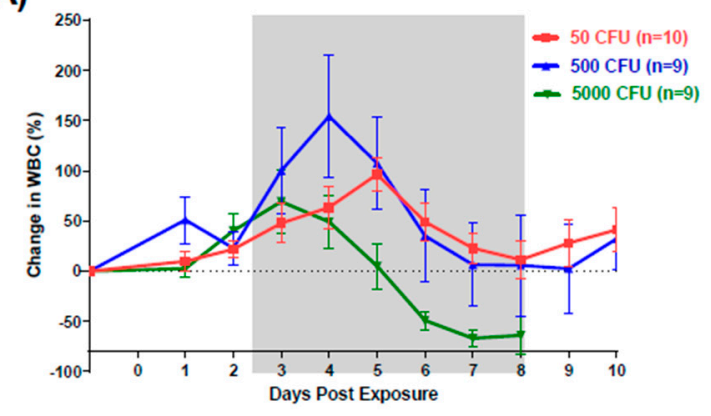

C)

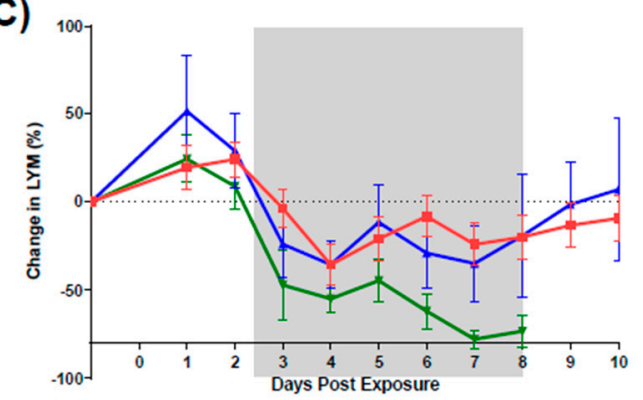

B)

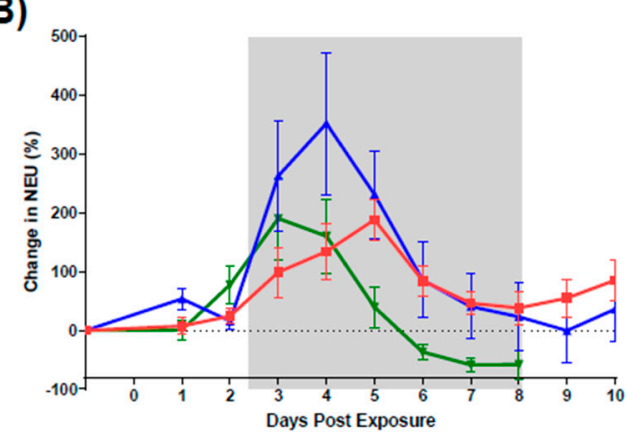

D)

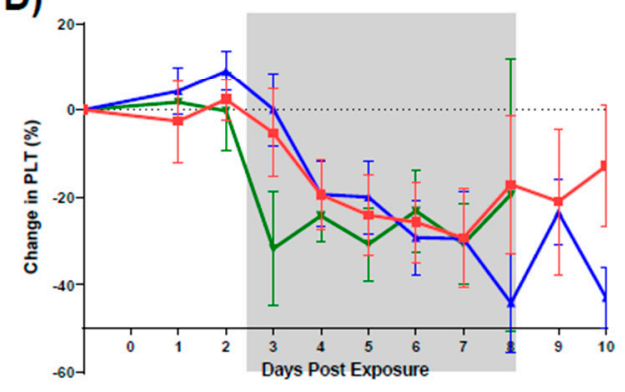

Figure 6. Changes in white blood cells after infection (suggested). Graphs show changes in average cell counts by dose group for (A) total white blood cells, (B) neutrophils, (C) lymphocytes, and (D) platelets.

\subsection{Pathology}

Postmortem examinations (necropsies) were performed on all euthanized macaques for macroscopic review with complete sets of tissues collected for microscopic analysis. The macroscopic lesions observed in this study were similar to those previously reported in cynomolgus macaques following aerosol exposure to F. tularensis SCHU4 regardless of the dose received, and / or the time-to-death following F. tularensis exposure. The most notable lesions were observed in the thoracic cavity, mainly in the lungs and mediastinal and tracheobronchial lymph nodes. 
A)

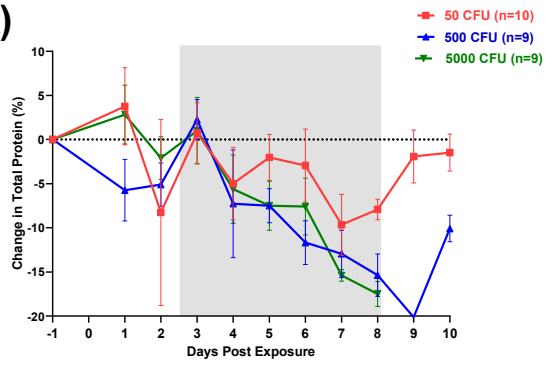

C)

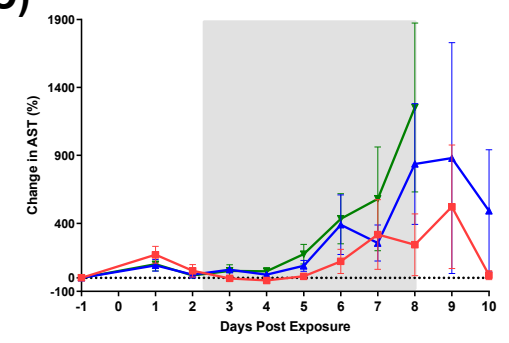

E)

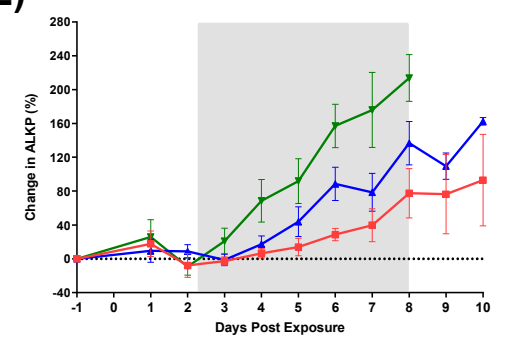

G)

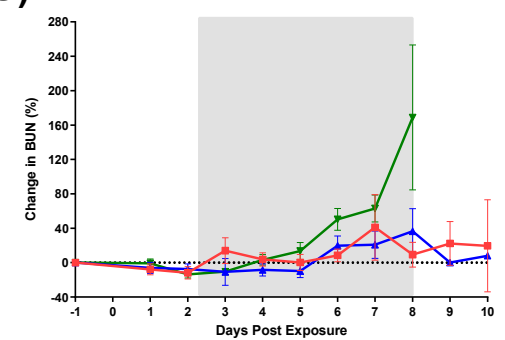

B)

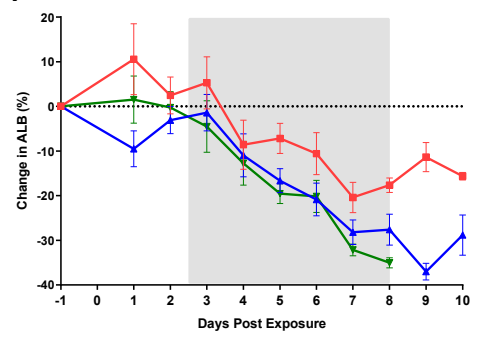

D)

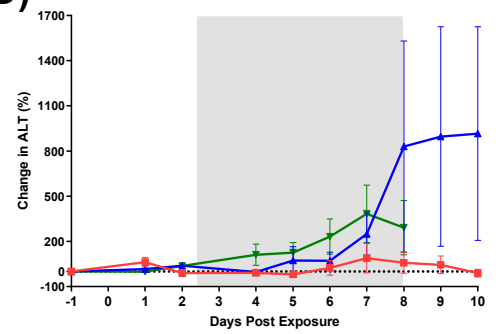

F)

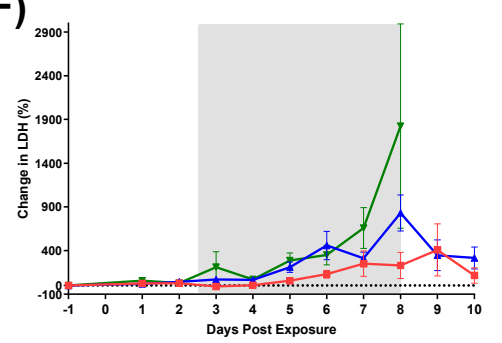

H)

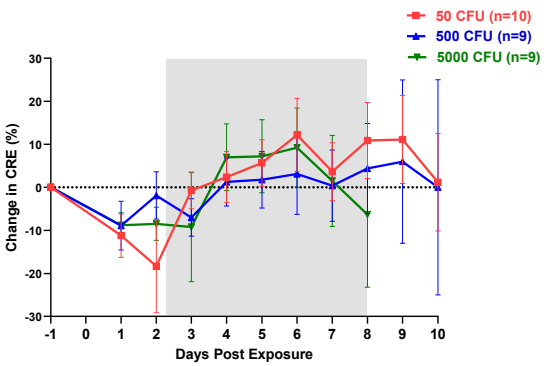

Figure 7. Changes in serum chemistry parameters in cynomolgus macaques exposed to different doses of aerosolized F. tularensis. (A) Total protein, (B) albumin, (C) aspartate transaminase (AST), (D) alanine transaminase (ALT), (E) alkaline phosphatase (ALKP), (F) LDH (lactate dehydrogenase), (G) blood urea nitrogen (BUN), (H) creatinine (CRE). Blood chemistries were analyzed with VITROS 250 chemistry analyzers.

During the gross postmortem examination, a focally extensive petechial rash (minimal in severity) was observed bilaterally in the axillary and inguinal areas of some of the macaques (Figure 8B). Macroscopic pulmonary changes varied in severity between macaques. Typically, all lung lobes were affected; however, lesions in the caudal (inferior) lung lobe were generally more severe. One or more of the following pulmonary changes were observed in the lung lobes: enlargement (failure to collapse); edema; mottled dark reddish-purple to black discoloration (consistent with severe pulmonary congestion, hemorrhage, and/or edema); and fibrinous pleuritis (a lesion seen more commonly on the pleural surface of the caudal (inferior) lung lobes). Fibrinous tags and adhesions loosely adhered to the thoracic wall and between the pleural surfaces of lung lobes, the diaphragm, and pericardial sac. Randomly scattered, well-circumscribed to coalescing, tannish-white, 
dome-shaped, firm to fluctuant foci (consistent appearance with necrosis) ranging in size from $6 \mathrm{~mm}$ up to $2 \mathrm{~cm}$ in diameter were prominent in the (caudal) lung lobes; however, similar lesions were randomly distributed in other lobes (Figure 8D).

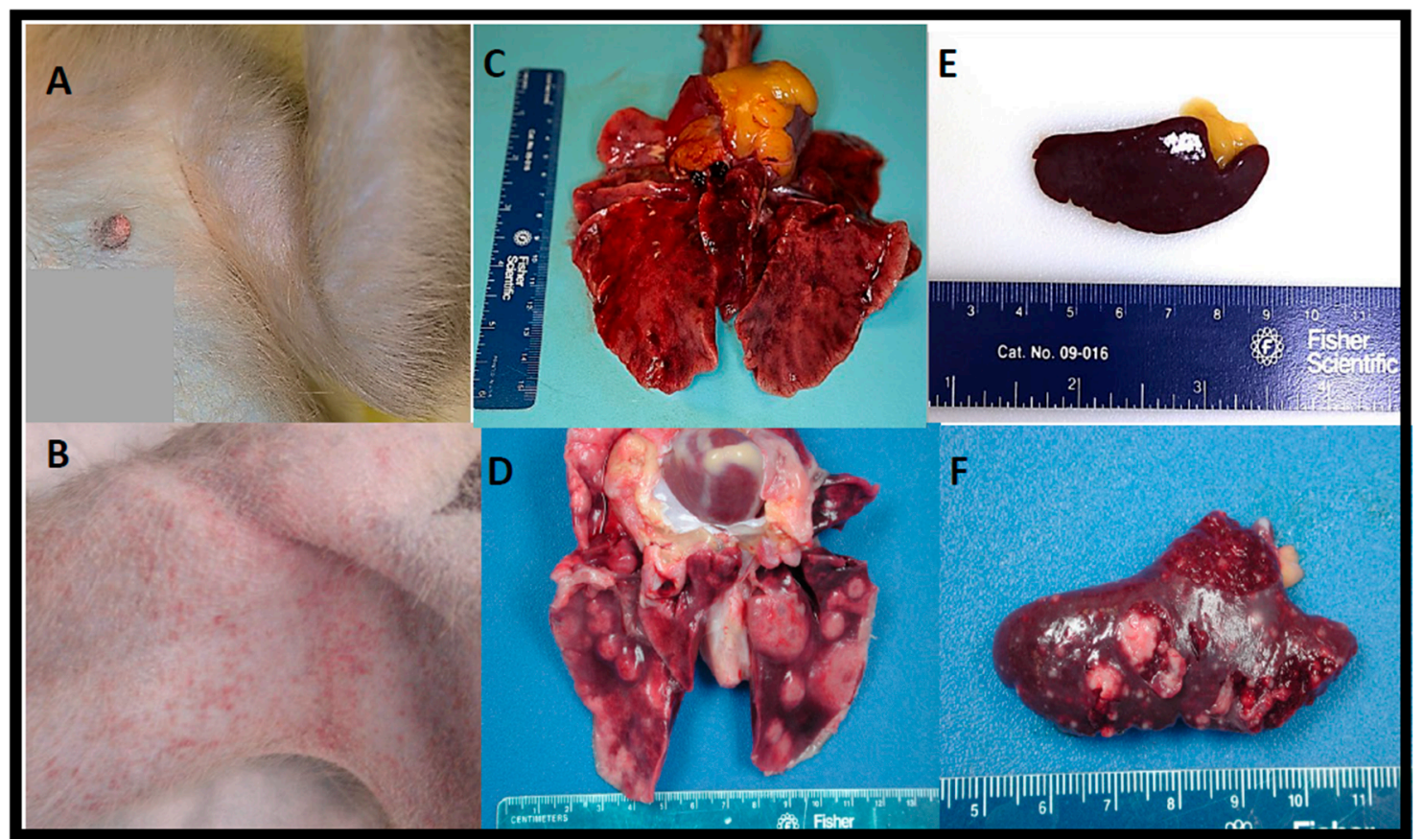

Figure 8. Comparison between nonchallenged control cynomolgus macaques and the gross pathological changes noted in cynomolgus macaques exposed to aerosolized F. tularensis. (A) Axillary area with no evidence of a cutaneous rash. (B) Axillary area of NHP exposed to 5000 CFU. (C) All lung lobes appear grossly normal with the exception of postmortem congestion. (D) Lung from a cynomolgus macaque exposed to 5000 CFU SCHU S4; hemorrhagic, edematous, and noncollapsing lung with multiple, raised necrotizing, and/or pyogranulomatous foci on all lung lobes. (E) Normal sized and shaped spleen. (F) Spleen from a cynomolgus macaque exposed to 50 CFU; multiple, tannish-white, flattened to slightly raised foci.

Enlargement (lymphadenopathy) of lymph nodes with edema and reddish discoloration (suggestive of hemorrhage/congestion) were the most common macroscopic findings. The mediastinal and tracheobronchial lymph nodes were the most commonly affected lymph nodes. However, similar findings were present in the mandibular, axillary, inguinal, and mesenteric lymph nodes. Splenic changes were also common but variable between macaques. The most noteworthy macroscopic lesions consisted of few to many (too numerous to count), up to $4 \mathrm{~mm}$ in diameter, tannish-white, flattened to slightly raised foci randomly dispersed throughout the parenchyma and capsular surface (Figure 8F). In some cases, a normal sized to moderately enlarged (splenomegaly), dark-reddish brown turgid spleen (suggestive of diffuse congestion and/or hemorrhage) was the sole splenic finding. For comparison, refer to Figure $8 \mathrm{~A}-\mathrm{C}$ for gross images of the axillary region, lung, and spleen, respectively, from a nonchallenged control cynomolgus macaque.

A mildly enlarged, dark reddish brown, congested liver (hepatomegaly) with an accentuated lobular pattern was observed in half of the macaques. Occasionally, randomly distributed, 1-2 mm tannish-white foci (consistent with necrosis) were seen on the hepatic surface; similar lesions were seen on the renal capsular surface of one macaque (data not shown).

Almost all macaques had noteworthy microscopic lesions specifically in the lung, lymph nodes, spleen, and bone marrow, which correlated well with the macroscopic findings described above. Lesions were similar in all macaques but varied in severity. Microscopic findings in the lungs consisted of necrotizing and suppurative bronchopneumonia (associated with larger conducting airways and arterioles) or randomly distributed 
vague to discrete inflammatory nodules (consistent with chronic abscesses and pyogranulomatous pneumonia) (Figure 9A-D). Intra-alveolar inflammation composed of neutrophils and macrophages were the earliest findings in all animals (Figure 9B). Macaques that expired at Days 7-9 post-exposure had lesions consistent with chronic abscesses, whereas those expiring at Days 14-17 dpi had more widespread pyogranulomatous inflammation (Figure 9C,D, respectively). Immunolabeled intra- or extracellular bacteria, bacterial fragments, and/or antigen were commonly seen in foci of necrosis in those tissues examined. Figure 9E,F show normal lung areas.

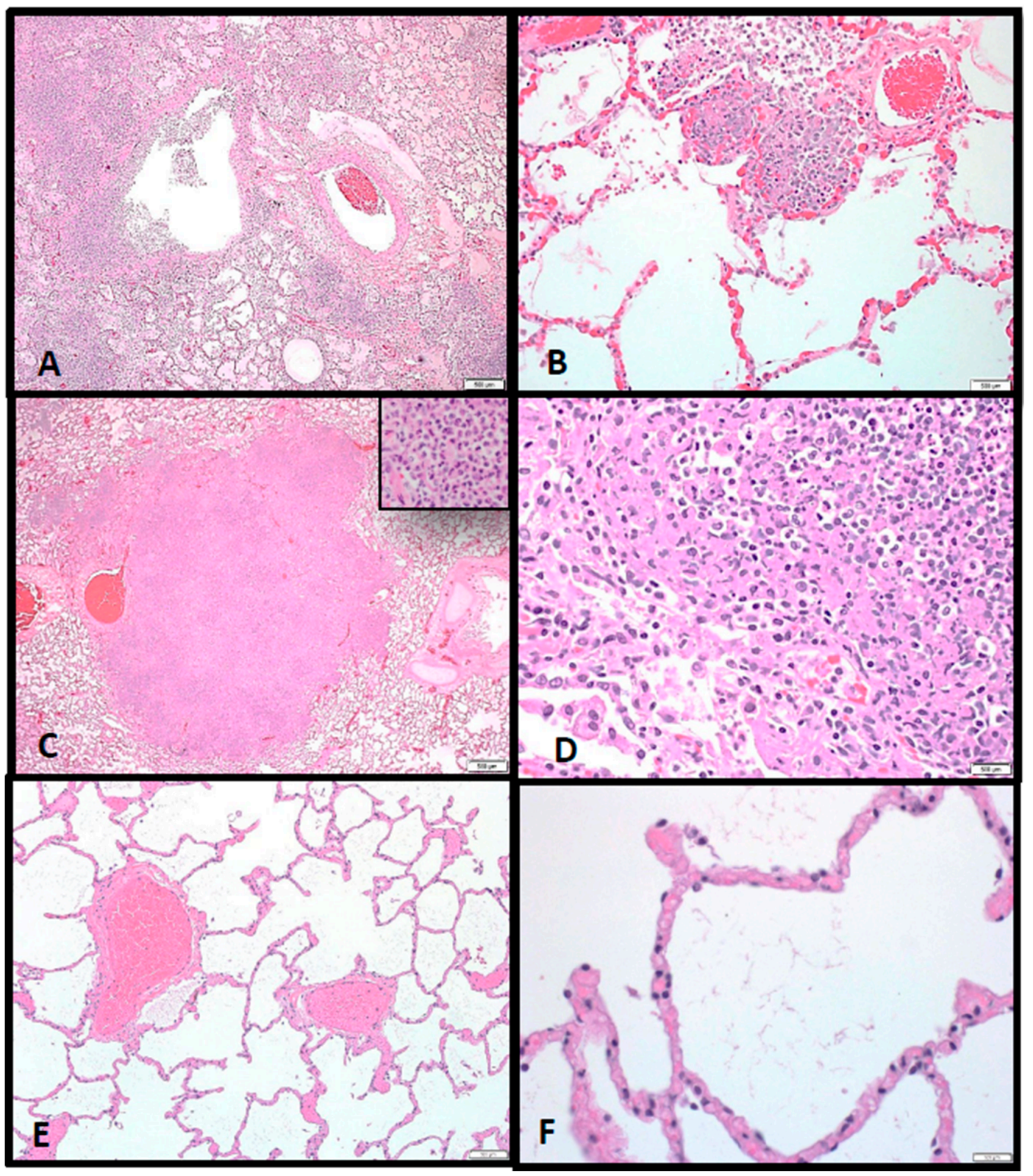

Figure 9. (A) Multifocal to coalescing foci of inflammation, often associated with larger conducting airways and arterioles, causing severe pulmonary consolidation (pneumonia), $2 \times$. (B) Alveolar inflammation composed of neutrophils and fewer macrophages is seen during initial infection, $10 \times$. (C) A well-circumscribed chronic abscess effaces pulmonary architecture in the 7-9 dpi animals, 20×; Inset. Chronic abscesses consist of degenerate neutrophils and fewer macrophages. (D) Abscesses become more widespread with significant pyogranulomatous inflammation characterized epithelioid macrophages with foamy vacuolated cytoplasm and fewer degenerate neutrophils at 14-17 dpi, 40×. (E) Normal alveolar airs spaces adjacent to arterioles from an aged match, nonchallenged NHP, $10 \times$. (F) No histologic evidence of inflammation present in the alveolar air spaces at higher magnification, $40 \times$. 


\section{Discussion}

Respiratory or inhalational infection with aerosolized F. tularensis in humans causes a rapid, severe bacterial pneumonia and sepsis which can be lethal with a minimum infectious dose of fewer than $10 \mathrm{CFU}$ [17]. Due to its high infectivity and relative ease of dissemination, F. tularensis has been studied as a potential candidate for weaponization and categorized as an agent of biological terrorism $[2,9,10]$. Development of medical countermeasures to lethal diseases such as inhalation tularemia is contingent on the characterization of animal models that effectively mimic the most severe form of disease in humans. The cynomolgus macaque has been established as the nonhuman primate model for pneumonic tularemia. To further characterize the disease in the cynomolgus macaque, groups of cynomolgus macaques were exposed to target doses of 50,500 or $5000 \mathrm{CFU}$ of aerosolized $F$. tularensis. Survival was dose dependent with target doses of $500 \mathrm{CFU}$ (134 to 749 CFU range) and 5000 CFU (1177 to 5860 CFU range) causing 100\% lethality by Days 7 and 8, respectively. Target doses of $50 \mathrm{CFU}$ (10 to $110 \mathrm{CFU}$ range) resulted in survival of 2 out of 10 macaques $(20 \%)$.

In humans, tularemia is an acute febrile illness. The disease manifestation is dependent on the route of infection, the dose, virulence of the strain, and host immune response. After an average incubation period of 3-5 days, there is an onset of symptoms including fever, chills, cough, headache, general malaise and in some cases diarrhea $[18,19]$. In this study, fever began in cynomolgus macaques generally between 2-3 after aerosol exposure to F. tularensis. Onset of other clinical changes noted (heart rate, blood pressure, changes in behavior, hematological changes) all coincided with fever onset. This data is strikingly similar to what has been reported in rats and rabbits, particularly with regard to fever onset and other clinical signs [20,21].

The generally mild increase in heart rate seen in most macaques was quite intriguing. While heart rate was elevated compared to baseline in 12 of 13 macaques, the heart rate in the febrile period was still within the normal range for an unrestrained adult cynomolgus macaque [22]. In other severe zoonotic infections in nonhuman primate models, heart rate increases between $40-60 \%$ in the febrile period (D.S. Reed, manuscript in preparation). This contrasts sharply with the median $20 \%$ increase seen here, and notably six of the thirteen macaques had a less than $20 \%$ increase in heart rate. Leibermeister's rule stipulates an $8 \mathrm{bpm}$ increase in heart rate for every $1 \mathrm{oC}$ elevation in body temperature [23]. This allowed us to calculate a predicted increase in heart rate during the febrile period; while 8 of 12 had an increase in heart rate greater than predicted, for four macaques (33\%) heart rate was lower than predicted. Intriguingly, Faget's sign (fever paired with bradycardia) has been reported in $42 \%$ of human tularemia patients [24]. Faget's has also been reported in yellow fever, typhoid, brucellosis, and Legionnaire's disease. This finding lends further credence to the cynomolgus macaque as a relevant model of human tularemia and would further suggest that this model could be used to gain a better understanding of the underlying pathophysiology associated with Faget's sign.

Hematology and clinical chemistry parameters in humans infected with F. tularensis have not shown any consistent remarkable findings. A review of 88 cases of tularemia from 1949 through 1979 reported increased white blood cell counts ranging from 5000 to 22,000 cells $/ \mathrm{mm}^{3}$ (median 10,400 cells $/ \mathrm{mm}^{3}$ ) upon hospital admission. An additional review of over 300 of cases reported that $50 \%$ presented with WBC counts between 3000 and 24,000 cells $/ \mathrm{mm}^{3}$ however the other half showed marked increases in leukocytes as great as 56,000 cells $/ \mathrm{mm}^{3}$ [22]. Similarly, in this study, there was a clear increase in WBC by Day 3 followed by a sharp decrease between Days 4 and 6 for all macaques; however, no group fell significantly outside of the normal range. This decrease was attributable to a loss in lymphocytes. A similar loss in platelets was also seen on Day 3. These changes in $\mathrm{CBC}$ match very well with what has been reported in both rats and rabbits. Increases in liver enzymes AST, ALT, and ALKP, as seen in this study, have been previously reported in human tularemia cases where of 58 patients tested, $58 \%$ had elevations in at least one of these three enzymes. 
As previously seen in lethal human cases of tularemia [25], in this study, the lung, lymph nodes (particularly the mediastinal and tracheobronchial lymph nodes), spleen, liver, and bone marrow were the most severely affected tissues during $F$. tularensis infection regardless of exposure dose. Although varying in severity, multifocal and coalescing, necrotizing and pyogranulomatous pneumonia and abscessation with (or without) extensive edema, fibrinous pleuritis, and pleural fibrosis were consistent pathological findings in almost all macaques and corresponded with the intrathoracic histopathologic changes. Additionally, necrotizing mediastinal lymphadenitis, splenitis, myelitis, and hepatitis were consistently observed regardless of the inhaled F. tularensis dose. The random focal necrotizing lesions in the liver were discrete but small unlike the larger coalescing lesions seen in the lung and spleen.

Here we demonstrated that aerosolized $F$. tularensis infection in cynomolgus macaques causes similar disease progression, pathogenesis, and clinical pathology profiles as compared to respiratory tularemia in humans. Therefore, the cynomolgus macaque model of pneumonic tularemia is an appropriate animal model for testing medical countermeasures for treatment of tularemia.

\section{Materials and Methods}

\subsection{Animals and Ethical Statement}

Twenty-eight healthy, adult female and male cynomolgus macaques (Macaca fascicularis) of primarily Chinese descent weighing between $3.6 \mathrm{~kg}$ and $7.4 \mathrm{~kg}$ were obtained from USAMRIID NHP colony. The age of the animals ranged from 3 years to 7 years. Animals were experimentally naïve with the exception of four animals that were either previously used as negative controls or survived a previous challenge with Francisella tularensis SCHU S4 and had a calculated exposure dose of 0 CFU with no signs of the disease and no antibodies against $F$. tularensis. Animals were in good physical condition, lacked physical malformations, and were free of clinical signs of infectious, contagious, or communicable diseases or parasites. All animals exposed to F. tularensis were handled in a BSL-3 containment laboratory at the U.S. Army Medical Research Institute of Infectious Diseases (USAMRIID). Research was conducted in compliance with the Animal Welfare Act and other federal statutes and regulations relating to animals and experiments involving animals, and adhered principles stated in the Guide for the Care and Use of Laboratory Animals, National Research Council, 1996. The facility where this research was conducted (USAMRIID) is fully accredited by the Association for the Assessment and Accreditation of Laboratory Animal Care International. Research was conducted under a protocol approved by the Institutional Animal Care and Use Committee (IACUC) at USAMRIID. All animals were examined and evaluated twice per day by study personnel. Early endpoint criteria, as specified by the score parameters within the "Post-exposure observations" section of these methods, were used to determine when animals should be humanely euthanized.

\subsection{Bacteria}

F. tularensis SCHU S4 strain (Biodefense and Emerging Infections Research Resources Repository (BEI Resources) Cat. No. NR-10492) was provided by the National Institute of Allergy and Infectious Diseases. A 25-mL flask of Mueller Hinton II (MHII) liquid medium containing $2 \%$ IsoVitaleX enrichment was inoculated with $10 \mu \mathrm{L}$ of a SCHU S4 seed stock. After $23 \mathrm{~h}$ of incubation at $37^{\circ} \mathrm{C}$ with shaking at $200 \mathrm{rpm}$, the optical density (OD660 nm) was measured. Logarithmic growth cultures were at the mid-logarithmic phase of growth and had an optical density (OD660 nm) of 0.15 to 0.85 . Microorganisms were diluted to the desired nebulizer concentration in MHII medium.

\subsection{Aerosol Exposures}

Each macaque was anesthetized by intramuscular (i.m.) injection of tiletamine/zolazepam $(3 \mathrm{mg} / \mathrm{kg})$ and challenged by aerosol as previously described [26]. Briefly, the respiratory function of each of the macaques was measured using whole-body plethys- 
mography (Buxco Systems, Sharon, CT) before aerosol challenge. Aerosol procedures were conducted using a 32-L, airtight Lexan chamber assembled in a head-only configuration for individual macaque exposures in a class III biological safety cabinet located inside a BSL-3 suite. All airflows, environmental monitoring, and system balancing were configured, controlled, and recorded through the institute standard automated bioaerosol exposure system (ABES)(Biaera Technologies, LLC, Hagerstown, MD [27]. Integrated air samples were obtained for each individual exposure using an all-glass impinger (AGI) drawing $6 \mathrm{~L} / \mathrm{min}$ from a port centered on the chamber and opposite to the animal's head. Aerosols were generated with a three-jet Collison nebulizer running at $7.5 \mathrm{~L} / \mathrm{min}, 25-30 \mathrm{psi}$. The amounts of live bacteria in AGI samples were analyzed on the same day as the aerosol exposure by performing a bacterial plating assay on Modified Thayer-Martin (MTM) agar plates to determine the colony forming units $(\mathrm{CFU}) / \mathrm{mL}$.

\subsection{Telemetry}

Macaques were implanted with a radiotelemetry device [Data Sciences International (DSI), St. Paul, MN] at least 14 days before aerosol exposure. Body temperatures, electrocardiography, and blood pressure were recorded once per hour by the DataQuest A.R.T. 4.1 system (DSI). Fever was defined as body temperature greater than two standard deviations above baseline temperature as determined by autoregressive integrated moving average (ARIMA) modeling. Due to telemetry equipment failure, two animals in the high target dose group had shorter baselines starting Day -1.5 and Day -0.5; for these animals, temperature recordings were initiated at approximately 12:00 PM on respective days.

\subsection{Post-Exposure Observations and Clinical Scoring}

Macaques were observed at least twice a day after aerosol exposure and scored for clinical signs of disease prior to, and while under anesthesia. The scoring parameters were as follows, appearance (0: normal with coat smooth, eyes/nose clear; 1: reduced grooming; 2: dull/rough coat, ocular/nasal discharge; 3: absence of grooming, or piloerection, or posture change), appetite (0: normal; 1 : decreased biscuit intake but still eats enrichment $\times 24 \mathrm{~h}$; 2 : will only eat food enrichment $\times 48 \mathrm{~h}$; 3: anorexic $\times 48 \mathrm{~h}$ ), clinical signs (0: normal temperature and respiration/min; 1 : Slight change in temperature/respiration $\left( \pm 1{ }^{\circ} \mathrm{C}\right.$ out of normal range $/<30 \% \pm n$ respiration range); 2 Temp: $\pm 2{ }^{\circ} \mathrm{C}$; respiration: $\pm 30 \%$ (above 51 or below 27); intermittent tremors; intermittent convulsions $<10$ min duration; transient prostration $<1 \mathrm{~h}$; 3 : Temp: $\pm 3{ }^{\circ} \mathrm{C}$; respiration: $\pm 50 \%$ (above 59 or below 19); continuous tremors or convulsions; persistent prostration $\geq 1 \mathrm{~h}$ ), natural behavior (0: normal; 1: minor changes, less interaction with staff; 2: little interaction, less mobile and alert, isolated; 3: no interaction, vocalization, self-mutilation, restless, or still), provoked behavior (0: normal; 1: subdued but normal when stimulated, 2: subdued even when stimulated, 3: unresponsive when stimulated, weak, pre-comatose). An extra point for each score of 3 was added to the cumulative clinical score. The early endpoint criteria for humane euthanasia, indicative of very poor health status, were cumulative clinical scores of 16-20 (maximum score), and/or a sudden drop of $>3{ }^{\circ} \mathrm{C}$ from baseline body temperature, continuous tremors or convulsions lasting $\geq 10$ min duration, persistent prostration lasting $\geq 2 \mathrm{~h}$, comatose or loss of consciousness. In some cases, such as accelerated dropping in body temperature without anesthesia, severe respiratory distress or continuous tremors, where the scoring range did not reflect the severity of the disease, the decision for euthanasia was made by the study director after consultation and verbal confirmation with the attending veterinarians.

\subsection{Clinical Laboratory Evaluation}

Blood samples were collected from central venous catheters (CVC) inserted in the internal jugular vein beginning fourteen days before, one day before, Days 1-21, and Day 28 after exposure. If collection from the CVC was not possible, blood samples were collected from the femoral vein of macaques anesthetized with $3 \mathrm{mg} / \mathrm{kg}$ intramuscular tile- 
tamine/zolazepam. Samples collected one day prior to exposure served as a reference baseline for each animal. Complete blood counts (CBCs) and blood chemistries were analyzed with Beckman Coulter hematology and VITROS 250 chemistry analyzers, respectively.

\subsection{Postmortem Examination (Necropsy)}

A full postmortem examination was performed under direct supervision of a pathologist board certified by the American College of Veterinary Pathologist in a BSL-3 necropsy facility. Tissues samples from all major organ systems (respiratory, gastrointestinal, genitourinary, hematopoietic, nervous, endocrine, skin and mucous membranes) were collected from each animal for histopathological and, in some cases, immunohistochemical examination. Tissue samples were frozen at $-70^{\circ} \mathrm{C}$ until processed for bacterial assays. All other tissues were immersion-fixed in $10 \%$ neutral buffered formalin for a minimum of 21 days prior to removal from biocontainment.

\subsection{Histology and Immunohistochemistry}

Formalin-fixed tissues for microscopic examination were trimmed, processed, and embedded in paraffin according to established protocols [28]. Embedded tissues were sectioned at 5-6 $\mu \mathrm{m}$ on a rotary microtome, mounted on glass slides, and stained with hematoxylin and eosin (H\&E). Tissues evaluated by immunohistochemistry were stained for F. tularensis lipopolysaccharide (LPS) (Meridian Life Science, Inc., Cincinnati, OH, USA) using a mouse monoclonal antibody (USAMRIID immuno \#927) and an immunoperoxidase assay system (EnVision System, DAKO Corp., Carpinteria, CA, USA). Unstained tissue sections were deparaffinized, rehydrated, subjected to methanol-hydrogen peroxide block, rinsed, and pretreated with proteinase $\mathrm{K}$ for $6 \mathrm{~min}$ at room temperature. A serum-free protein block (DAKO) plus 5\% normal goat serum was applied for $30 \mathrm{~min}$. The monoclonal antibody was then applied to the tissue at a dilution of 1:1200 and incubated at room temperature for $60 \mathrm{~min}$. The tissue was exposed to the EnVision horseradish peroxidase labeled polymer for $30 \mathrm{~min}$ at room temperature. All sections were exposed to 3,3'diaminobenzidine $(\mathrm{DAB})$ permanent chromogen for $5 \pm 1 \mathrm{~min}$, rinsed, counter-stained with hematoxylin, dehydrated, and cover-slipped with Permount. Normal uninfected lung tissue served as the negative control; the positive control tissue was lung tissue from a known F. tularensis-infected monkey. Normal mouse IgG was used as the negative serum control for the control slides.

\subsection{Statistical Analysis}

Survival curves were calculated by the Kaplan-Meier product-limit method. Body temperatures were recorded by telemetry once per hour, beginning at least $12 \mathrm{~h}$ and up to 3 days prior to exposure and continuing up to 28 days post-exposure. Pre-exposure temperature data taken between two to five days pre-exposure was used to develop a baseline period to fit an ARIMA model. Forecasted values for the post-exposure periods were based on the baseline extrapolated forward in time using SAS ETS (version 9.3). For body temperature, residual changes greater than two standard deviations were used to compute fever duration (number of hours of significant temperature elevation), fever hours (sum of the significant temperature elevations), and average fever elevation (fever hours divided by fever duration in hours). The number of surviving animals in each group was statistically insufficient at later time points; therefore, only post-exposure data through Day 8 was used for analysis. T-tests with stepdown bootstrap adjustment were utilized to compare mean temperature variables between groups. ANOVA at each individual time point was completed with post hoc Tukey's tests for pairwise comparisons for respiratory rates, CBCs and chemistries for time points Day 0 through Day 8. Beyond time point Day 8, there were insufficient samples for comparison. For analysis of ECG and blood pressure data, data were imported into Ponemah 6.5 (DSI) and analyzed using MatLab 2020a and GraphPad software. 
Supplementary Materials: The following are available online at https:/ / www.mdpi.com/article/10 .3390/ pathogens10050597/s1, Table S1: No significant differences in fever between dose groups.

Author Contributions: Data curation, S.L.N., D.S.R., A.N.; formal analysis, O.M.F., V.A.L., C.A.W., S.L.N., D.A.A., P.R.F., D.S.R., A.N.; funding acquisition, A.N.; investigation, O.M.F., V.A.L., D.A.A., D.S.R., A.N.; methodology, O.M.F., V.A.L., D.A.A., D.S.R., A.N.; project administration, O.M.F., V.A.L., D.A.A., A.N.; resources, O.M.F., V.A.L., D.A.A., A.N.; supervision, A.N.; visualization, O.M.F., D.A.A., P.R.F., D.S.R., A.N.; writing-original draft, O.M.F., C.A.W., D.A.A., D.S.R., A.N. All authors have read and agreed to the published version of the manuscript.

Funding: This study was supported by an interagency agreement between Office of Biodefense, Research Resources and Translational Research (OBRRTR)/National Institute of Allergy and Infectious Diseases (NIAID) and USAMRIID. Funding for Dr. Reed was provided by the National Institutes of Health, grant number 1 R01 AI123129-01.

Institutional Review Board Statement: These experiments and procedures were reviewed and approved by the United States Army Medical Research Institute for Infectious Diseases (USAMRIID) Institutional Animal Care and Use Committee (IACUC) on 29 January 2009. All research (Project \# 92577) was conducted in compliance with the USDA Animal Welfare Act (PHS Policy) and other federal statutes and regulations relating to animals and experiments involving animals and adheres to the principles stated in the Guide for the Care and Use of Laboratory Animals, National Research Council, 2011. The facility is fully accredited by the Association for Assessment and Accreditation of Laboratory Animal Care, International.

Data Availability Statement: Data available on request due to restrictions and clearance processes in USAMRIID.

Acknowledgments: We thank the personnel in the Aerosol Services Branch of the Veterinary Medicine Division for conducting the aerosol sprays of animals, the personnel of the Veterinary Medicine Division for the care and handling of the animals and the Clinical Lab personnel for performing hematology and chemistry tests in these studies. Special thanks to Pathology Division's Stephen Akers, Neil Davis, Gale Kreitz and Chris Mech for providing necropsy, histochemical and immunohistochemical support. Furthermore, we gratefully acknowledge Diana Fisher for her assistance with statistics. We also thank Judy Hewitt (NIAID) for excellent discussion and guidance during this study and Tina Guina, Lynda Lanning and Mark Williams (NIAID) for critically reviewing the data and manuscript.

Conflicts of Interest: The authors declare no conflict of interests.

\section{References}

1. Nigrovic, L.E.; Wingerter, S.L. Tularemia. Infect. Dis. Clin. N. Am. 2008, 22, 489-504. [CrossRef]

2. Dennis, D.T.; Inglesby, T.V.; Henderson, D.A.; Bartlett, J.G.; Ascher, M.S.; Eitzen, E.; Fine, A.D.; Friedlander, A.M.; Hauer, J.; Layton, M.; et al. Working Group on Civilian B., Tularemia as a biological weapon: Medical and public health management. JAMA 2001, 285, 2763-2773. [CrossRef] [PubMed]

3. Hepburn, M.J.; Friedlander, A.M.; Dembek, Z.F. Tularemia. In Medical Aspects of Biological Warfare; Lenhart, M.K., Ed.; Borden Institute: Washington, DC, USA, 2007; pp. 167-184.

4. Helvaci, S.; Gedikoglu, S.; Akalin, H.; Oral, H.B. Tularemia in Bursa, Turkey: 205 cases in ten years. Eur. J. Epidemiol. 2000, 16, 271-276. [CrossRef] [PubMed]

5. Komitova, R.; Nenova, R.; Padeshki, P.; Ivanov, I.; Popov, V.; Petrov, P. Tularemia in Bulgaria 2003-2004. J. Infect. Dev. Ctries. 2010, 4, 689-694. [CrossRef]

6. Reintjes, R.; Dedushaj, I.; Gjini, A.; Jorgensen, T.R.; Cotter, B.; Lieftucht, A.; D'Ancona, F.; Dennis, D.T.; Kosoy, M.A.; MulliqiOsmani, G.; et al. Tularemia outbreak investigation in Kosovo: Case control and environmental studies. Emerg. Infect. Dis. 2002, 8,69-73. [CrossRef]

7. Steiner, D.J.; Furuya, Y.; Metzger, D.W. Host-pathogen interactions and immune evasion strategies in Francisella tularensis pathogenicity. Infect. Drug. Resist. 2014, 7, 239-251.

8. Feldman, K.A.; Enscore, R.E.; Lathrop, S.L.; Matyas, B.T.; McGuill, M.; Schriefer, M.E.; Stiles-Enos, D.; Dennis, D.T.; Petersen, L.R.; Hayes, E.B. An outbreak of primary pneumonic tularemia on Martha's Vineyard. N. Engl. J. Med. 2001, 345, $1601-1606$. [CrossRef] [PubMed]

9. Teutsch, S.M.; Martone, W.J.; Brink, E.W.; Potter, M.E.; Eliot, G.; Hoxsie, R.; Craven, R.B.; Kaufmann, A.F. Pneumonic tularemia on Martha's Vineyard. N. Engl. J. Med. 1979, 301, 826-828. [CrossRef]

10. Alibek, K. Biohazard; Random House, Inc.: New York, NY, USA, 1999; pp. 25-27. 
11. Kortepeter, M.G.; Parker, G.W. Potential biological weapons threats. Emerg. Infect. Dis. 1999, 5, 523-527. [CrossRef] [PubMed]

12. Thomas, L.D.; Schaffner, W. Tularemia pneumonia. Infect. Dis. Clin. N. Am. 2010, 24, 43-55. [CrossRef] [PubMed]

13. Oyston, P.C.; Sjostedt, A.; Titball, R.W. Tularaemia: Bioterrorism defence renews interest in Francisella tularensis. Nat. Rev. Microbiol. 2004, 2, 967-978. [CrossRef]

14. Lyons, R.C.W.; Terry, H. Animal models of Francisellla tularensis infection. In Francisella tularensis: Biology, Pathogenicity, Epidemiology, and Biodefense; Abu Kwaik, Y., Metzger, D.W., Nano, F., Sjöstedt, A., Titball, R., Eds.; The New York Academy of Sciences: New York, NY, USA, 2007; pp. 238-265.

15. Glynn, A.R.; Alves, D.A.; Frick, O.; Erwin-Cohen, R.; Porter, A.; Norris, S.; Waag, D.; Nalca, A. Comparison of experimental respiratory tularemia in three nonhuman primate species. Comp. Immunol. Microbiol. Infect. Dis. 2015, 39, 13-24. [CrossRef]

16. Guina, T.; Lanning, L.L.; Omland, K.S.; Williams, M.S.; Wolfraim, L.A.; Heyse, S.P.; Houchens, C.R.; Sanz, P.; Hewitt, J.A. The Cynomolgus Macaque Natural History Model of Pneumonic Tularemia for Predicting Clinical Efficacy under the Animal Rule. Front. Cell. Infect. Microbiol. 2018, 8, 1-17. [CrossRef] [PubMed]

17. Saslaw, S.; Eigelsbach, H.T.; Prior, J.A.; Wilson, H.E.; Carhart, S. Tularemia vaccine study. II. Respiratory challenge. Arch. Intern. Med. 1961, 107, 702-714. [CrossRef] [PubMed]

18. Dienst, F.T., Jr. Tularemia: A perusal of three hundred thirty-nine cases. J. La. State. Med. Soc. 1963, 115, 114-127. [PubMed]

19. Stuart, B.M.; Pullen, R.L. Typhoid; clinical analysis of 360 cases. Arch. Intern. Med. 1946, 78, 629-661. [CrossRef] [PubMed]

20. Hutt, J.A.; Lovchik, J.A.; Dekonenko, A.; Hahn, A.C.; Wu, T.H. The Natural History of Pneumonic Tularemia in Female Fischer 344 Rats after Inhalational Exposure to Aerosolized Francisella tularensis Subspecies tularensis Strain SCHU S4. Am. J. Pathol. 2017, 187, 252-267. [CrossRef] [PubMed]

21. Reed, D.S.; Smith, L.; Dunsmore, T.; Trichel, A.; Ortiz, L.A.; Cole, K.S.; Barry, E. Pneumonic tularemia in rabbits resembles the human disease as illustrated by radiographic and hematological changes after infection. PLoS ONE 2011, 6, e24654. [CrossRef]

22. Gauvin, D.V.; Tilley, L.P.; Smith, F.W., Jr.; Baird, T.J. Electrocardiogram, hemodynamics, and core body temperatures of the normal freely moving cynomolgus monkey by remote radiotelemetry. J. Pharmacol. Toxicol. Methods 2006, 53, 140-151. [CrossRef]

23. Shah, B.S. Mirror-Image Relationship between Temperature and Pulse. JAMA 1979, 242, 2760-2761. [CrossRef]

24. Evans, M.E.; Gregory, D.W.; Schaffner, W.; McGee, Z.A. Tularemia: A 30-year experience with 88 cases. Medicine 1985, 64, 251-269. [CrossRef] [PubMed]

25. Evans, M.E. Francisella tularensis. Infect. Control. 1985, 6, 381-383. [CrossRef] [PubMed]

26. Dabisch, P.A.; Kline, J.; Lewis, C.; Yeager, J.; Pitt, M.L. Characterization of a head-only aerosol exposure system for nonhuman primates. Inhal. Toxicol. 2010, 22, 224-233. [CrossRef] [PubMed]

27. Hartings, J.M.; Roy, C.J. The automated bioaerosol exposure system: Preclinical platform development and a respiratory dosimetry application with nonhuman primates. J. Pharmacol. Toxicol. Met. 2004, 49, 39-55. [CrossRef] [PubMed]

28. Prophet, E.B.; Mills, B.; Arrington, J.B.; Sobin, L.H. Laboratory Methods for Histotechnology. 1992. Available online: https: / / babel.hathitrust.org/cgi/pt?id=mdp.39015029715979\&view=1up\&seq=8 (accessed on 22 February 2021). 